\title{
MEMS Based Position and Motion Sensors for Controlling Complex Parallel Mechanisms
}

\author{
Tesfay Tesfu Mekonnen, Josef Schlattmann \\ Hamburg University of Technology \\ Institute of Laser and system Technologies \\ Denickestrsse 17 \\ 21073 Hamburg
}

\section{Introduction}

In order to effectively control motion in machines, measurements of positions, displacements, tilts, velocities, accelerations, vibrations, etc. are necessary. Sensors employed to measure such parameters have to be optimally selected and integrated based on given applications in order to assure efficient, accurate, and reliable functioning of the machine system. The general objective of this application research is to develop and validate sensor based techniques for real time pose determination and control of parallel mechanisms. Specifically, it deals with the implementation of stable walking of the biped robot Centaurob [Fig.1]. For the stability control, motion sensors are to be selected systematically.
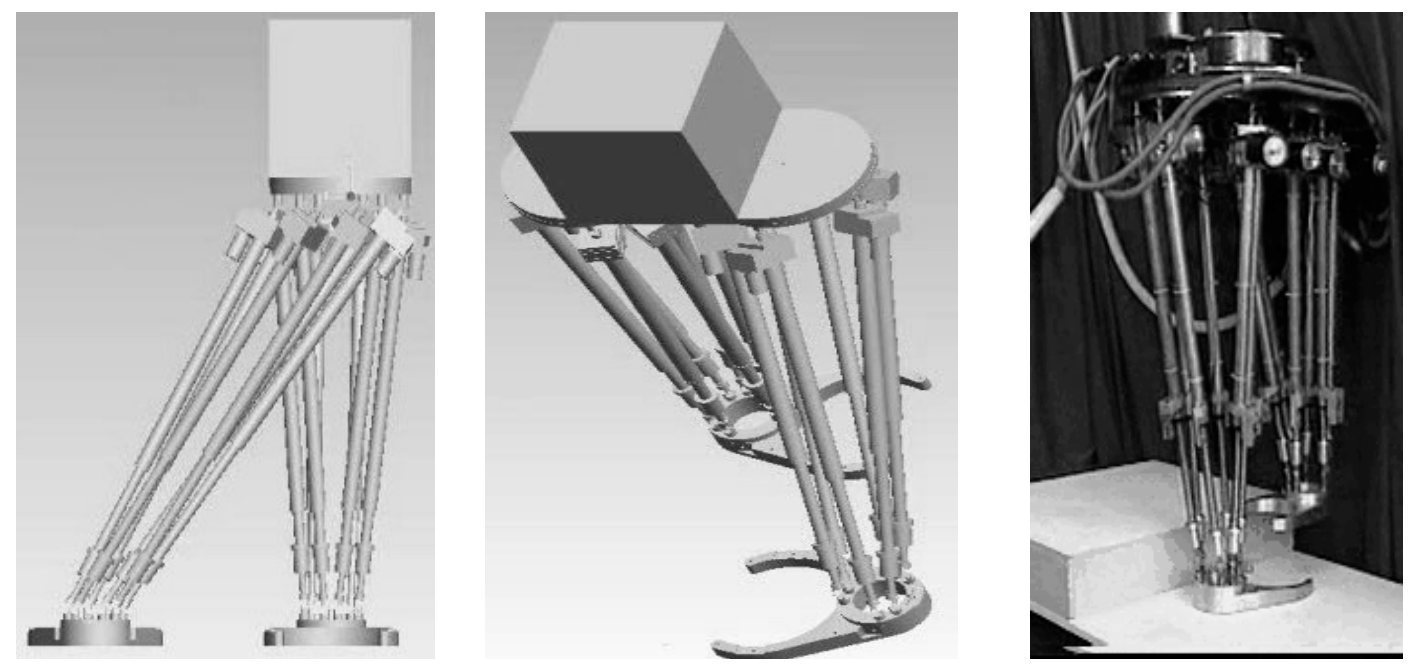

Fig. 1 Statically stable walking machine Centaurob

Centaurob is a statically stable walking robot, whose basic working principle was successfully verified in a previous research work [1]. The basic mechanical structure of Centaurob is made up of two serially coupled Stewart platforms (hexapods). The position and orientation in space (pose) of the robot have to be determinable using selected motion sensors. In hexapod structure the control variables are the lengths of the actuator links. But since solving forward kinematic equations of motion for such parallel mechanisms is not deterministic, it is not possible to compute the pose of moving platforms in absolute terms based on the lengths. Therefore, sensor-based solution methods are to be employed to compute the robot's forward kinematics and hence pose. Because of a stringent size/shape and precision requirements, it is challenging to find a fitting sensor solution. In order to identify the measured parameters, first a mathematical model of static walking robot has to be developed. In the following sections, the robot control model approaches and involved problems will be briefly discussed followed by a systematic motion sensor selection and integration methods.

\section{The solution goals}

The goal is to develop an accurate, reliable, future oriented and cost effective sensor solution for real time controlling of parallel mechanisms in general and the biped robot Centaurob, in particular. The number of sensors are to be kept as minimum as possible. Minimizing the number of sensors saves IO space on the controller, which is a scarce resource in a complex control system. Given a fast computer, non measured 
parameters can be computed in real time from measured sensor information. Here, it is important to note that the computed parameters inherit inaccuracies from the measured parameters, leading to error propagation. On the other hand if the measured parameters are arranged serially, the reliability of the overall system has to be considered carefully. In such scenarios a lot of weight has to be put on the reliability and accuracy of individual sensors. Centaurob has a very limited space left for sensors making size and shape other critical parameters. Moreover, the sensors operate with and around servomotors meaning electromagnetic noise interference from the environment is high. Technology-wise, mainly MEMS based motion sensors are to be explored thoroughly.

\section{Statically stable walking application of Centaurob}

Fig. 2 and Fig. 3 below show the walking technique of Centaurob schematically. The balance function of Centaurob is implemented in computers using different algorithms [2-3]. Basically the computer receive data from motion sensors, process these data using preprogrammed balance control algorithms, and commands each actuator, according to the instability computed by the algorithm. Selection and integration of appropriate motion sensors is, therefore, a very important step in development of balance control system. Before specific sensors can be selected, the robot control solution approach has to be developed. The objective here is to develop methods for accurate and reliable measurement of a pose of moving platform for parallel kinematic structures. Detailed definition of the solution method is important because the parameters used in selecting sensors depend greatly on the solution e.g. type, accuracy, size/shape, etc of the sensor. A mathematical model of the selected solution approach is the bases for defining the parameter values such as accuracy, size, number of sensors required, etc. Position ( $x, y, z$ coordinate) and orientation (pitch, roll, and yaw angle) computing algorithms are also developed on the bases of the mathematical model.

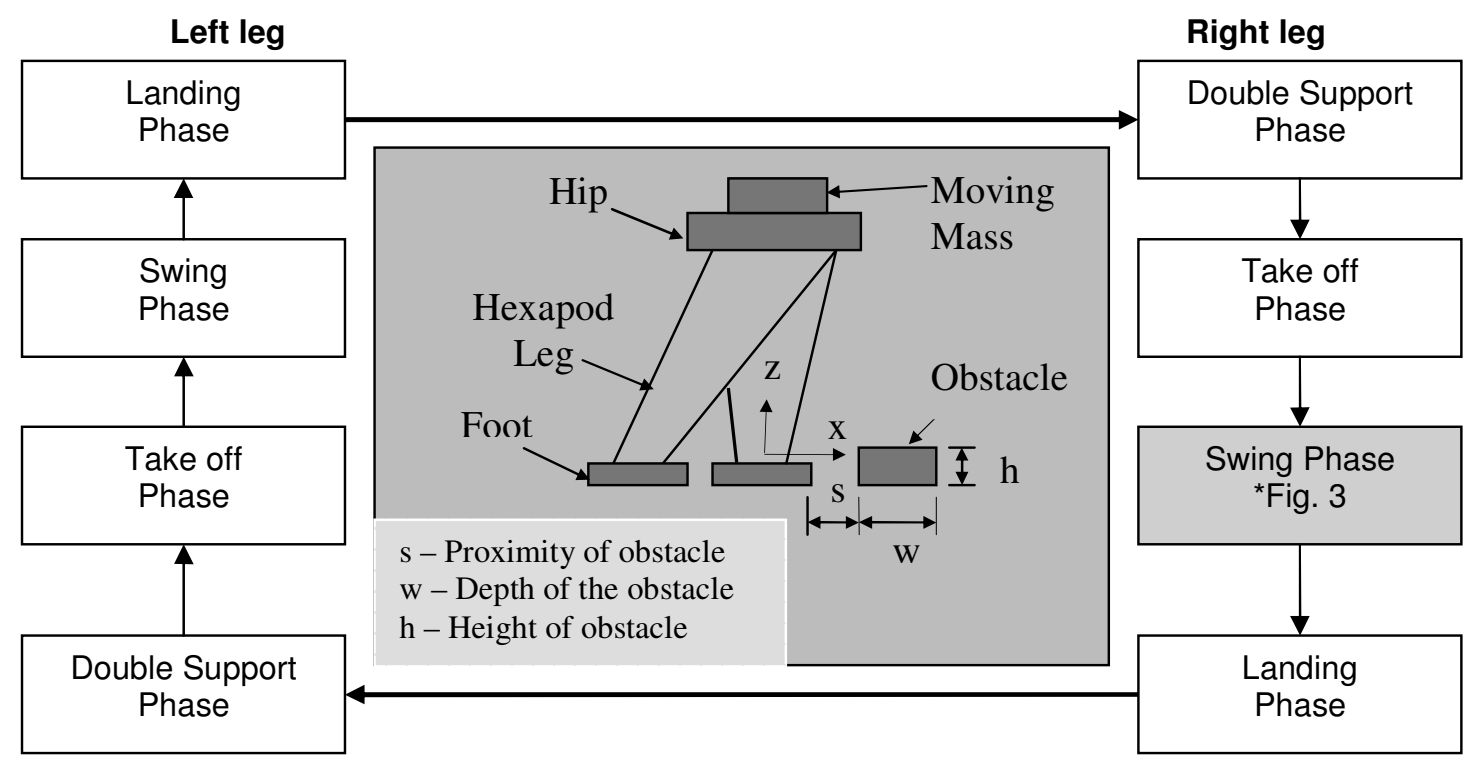

Fig.2 Walking phases and obstacle
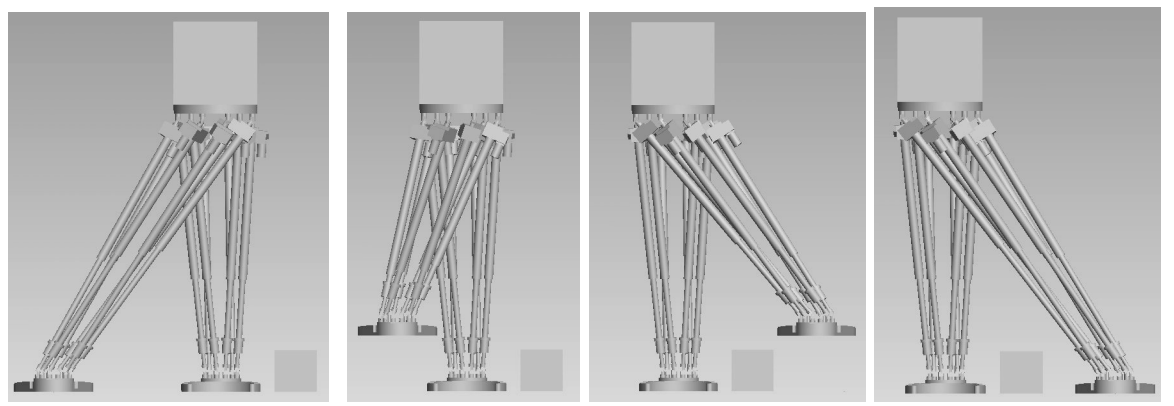

*Fig.3 States of right leg swing phase 
The specific purpose of the motion sensors in this application is to track the centre of mass of a biped robot in order to implement a balanced walking. In addition, the motion sensors should be able to compute the position of the swing foot in real time. This can be accomplished in a number of different ways. For example, a pose of a ground fixed hexapod can be deterministically computed using one of the following three approaches.

- two dual axis inclination sensors and a length sensor [Fig. 4]

- three length sensors and a gyroscope

- two length sensors, a three axis gyroscope, and an angle sensor

In order to deterministically compute a pose of Centaurob, the position and orientation of the components indicated in light color in Fig. 4 are to be deterministically localized.
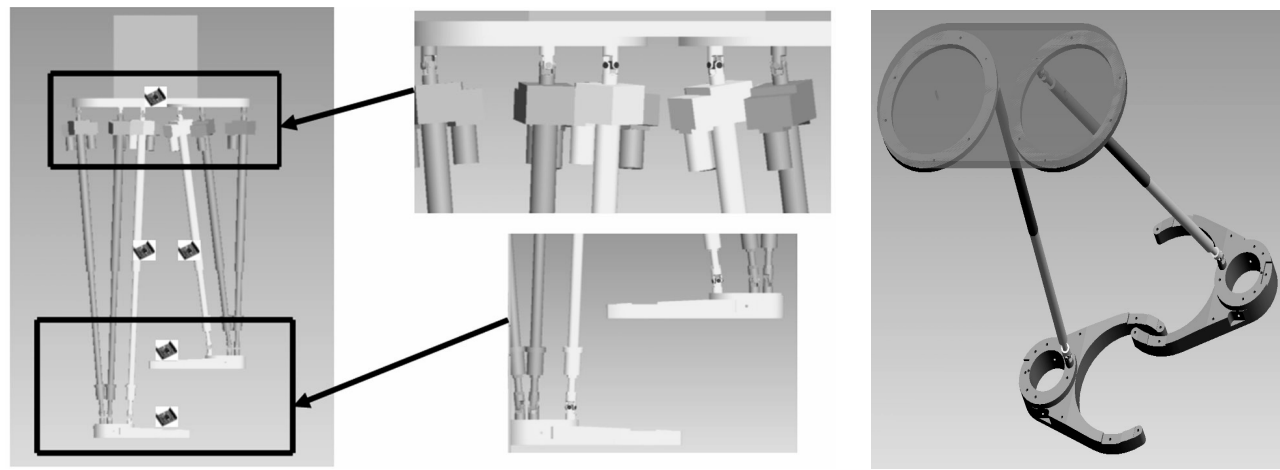

Fig. 4 Structural components to be absolutely localized using motion sensors
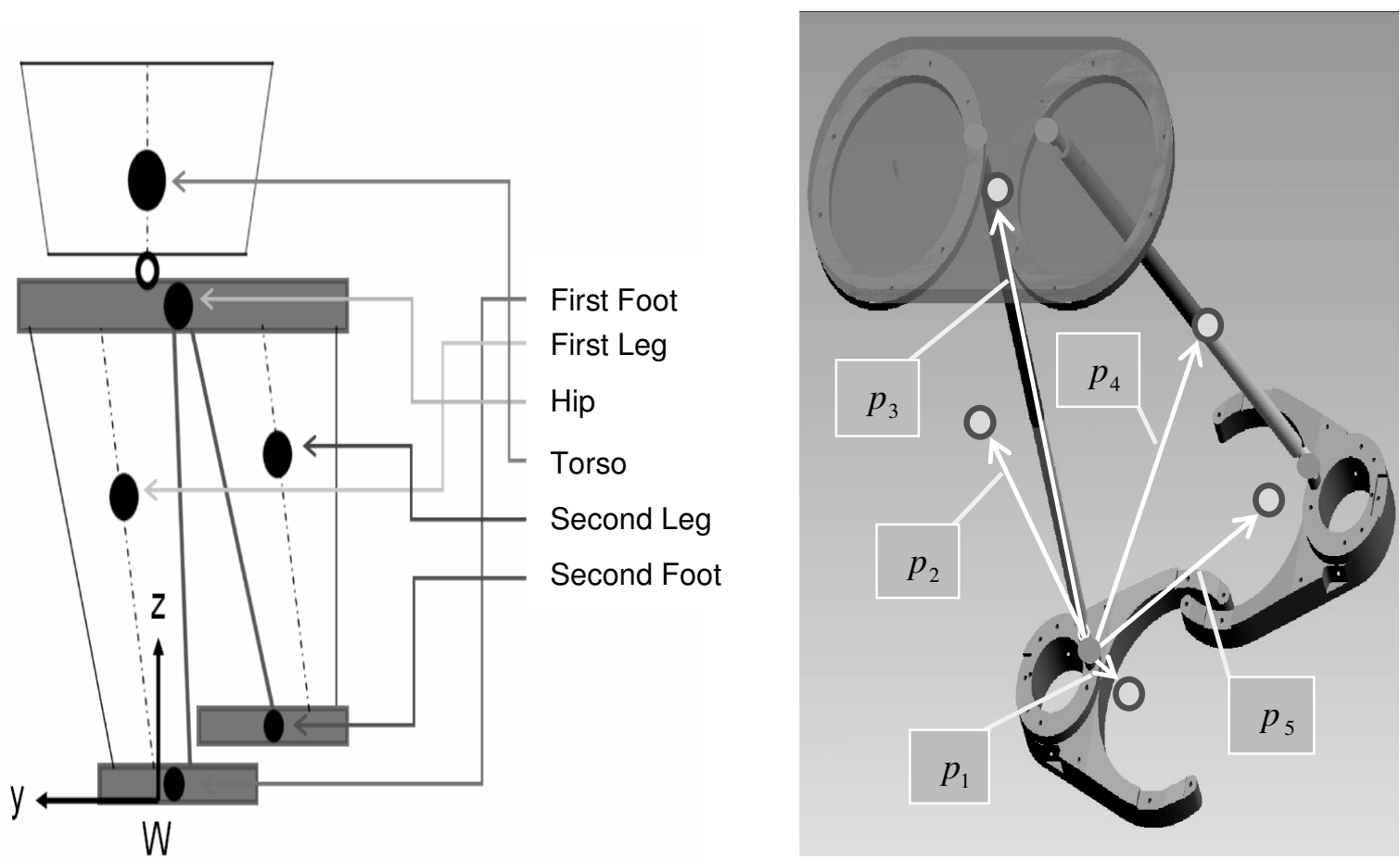

Fig. 5 Centre of mass points of Centaurob links

For the purpose computing body orientation, appropriate angular position/displacement sensors, linear position/displacement sensors, accelerometers, inclinometers and/or gyroscopes have to be selected. The data collected from sensors allows a computer to track the position and orientation of bodies using pre-specified algorithms. The known and measurable variables are determined based on the mathematical equation describing the objective functions. The main objective functions in Centaurob control application are the centre of mass (CoM) of the robot and the position vector of the swing foot. In order to be able to compute the CoM of the robot the positions of the centre of mass of each link $(\mathrm{Pi})$ of 
the system should be known. For the purpose of defining centre of mass of the robot, a reduced model of Centaurob is developed as shown in Fig. 5. In this reduced model the centre of mass of the hexapod-links of each leg are approximated with a single mass lying on the centre line of each hexapod respectively. CoM can then be computed as:

$$
\begin{aligned}
C o M= & \frac{\sum_{i} m_{i} p_{i}}{\sum_{i} m_{i}} ; \text { Where: } \\
& m_{i} \text { - Mass of the } \mathrm{i}^{\text {th }} \text { link } \\
& p_{i} \text { - Position of the centre of mass of } \mathrm{i}^{\text {th }} \text { link }
\end{aligned}
$$

After analyzing the mechanisms thoroughly, it is decided to solve the application using combinations of tilt and displacement sensors. Tilt sensors are for computing the orientation in space of selected robot structures. Displacement sensors are for measuring the variable lengths of the hexapod links. In order to deterministically compute the pose of a given component in a parallel kinematic structure whose links are connected with universal joints, the measurements of four angles and one displacement/length are required. This can be performed using two dual axis tilt sensors and one linear position sensor. Measurement of body orientation for a moving body usually faces limitations in accuracy.

When a robot is initialized from power-off state, the sensor system should be able to determine the exact position and pose of each part of the robot without the need of complicated calibration process. This means the robot requires absolute measurements of linear positions and angles. The need to select an absolute sensor is especially evident in the case of the hexapod link length measurement. An absolute platform orientation with respect to Earth's gravity vector can be measured using dual axis inclination sensors. Most of acceleration based tilt sensors deliver absolute roll and pitch angle. In order to measure absolute rotation in a horizontal plane i.e. yaw angle, a gyroscope can be used. Gyroscopes are usually employed in combination (in fusion) with accelerometers e.g. inertial measurement systems.

\section{Sensor selection and integration}

Sensors are by nature very diverse system components both functionally and constructionwise. Because of too many competing motion sensors in the market, it is easy to loose sight in the search process, if the selection process is not done in a systematic way. The variation in cost and quality can be huge. To avoid painful problems at later stage, it is recommended to make sensor design and/or selection in a systematic way. Depending on the type and application of a given sensor, various parameters play a role in its selection and evaluation. Size or dimension, orientation, direction of movement, portability, compatibility with other hardware and software components, analog or digital, types of interfaces (RS232, PWM, CAN, etc), incremental or absolute, power supply, wear and tear, ease of installation, ease of calibration, measurement range, measurement frequency, resolution, accuracy, dynamic behavior, operating environment, signal sensitivity, noise sensitivity, price, availability, technological trend, etc. could all play a role to some extents. Thorough analysis and understanding of the sensor application is, therefore, necessary in order to be able to make informed tradeoffs among different influence parameters in the selection process. Here, one can follow a methodical evaluation methodology that employs systematic scoring and ranking of different sensor solutions against weighted evaluation criteria [5-6].

MEMS sensors are evolving as a cost effective substitutes for traditional electromechanical sensors [4]. This paper, therefore, specifically deals with MEMS based sensor solutions technology-wise. MEMS inclinometers/accelerometers are, for example generally, smaller, lighter, more reliable, more functional, and cheaper, in comparison to their conventional counterparts. They are also compatible with other trends in sensor technology. The most noticeable technology trends in measurement and control systems are advances in wireless technologies, FPGAs, USB data acquisitions (using laptops), etc. The performances of ADCs are also improving while their prices declining. Due to the possibility of integrating application specific signal preparation and processing functions into MEMS cores, the MEMS sensors can be installed close at the measurement location. Wireless data transmission can be employed both to save space and minimize electromagnetic interferences. MEMS applications are not without a challenge, however. One of the most evident challenges is lack of standardized packaging with established quality standards. Moreover, highly specialized and expensive fabrication and prototyping technology makes it less accessible for sensor designers. Now the problem is reduced to selecting specific sensors fulfilling the stringent requirements discussed above. 


\section{The control system testing tools}

For developing and testing the selected sensor solutions, a PC-based hardware-in-the-loop (HiL) simulation method is being used. Here, Matlab/Simulink/Stateflow graphical development tools and dSPACE real time control system are used to interact with various sensors and actuators. The dSPACE controller board posses different types of sensor interfaces such as ADC, Digital I/Os, PWM, RS232, RS422/RS485, Encoder inputs, etc. and it works with Matlab/Simulink/Stateflow models seamlessly [7-8].

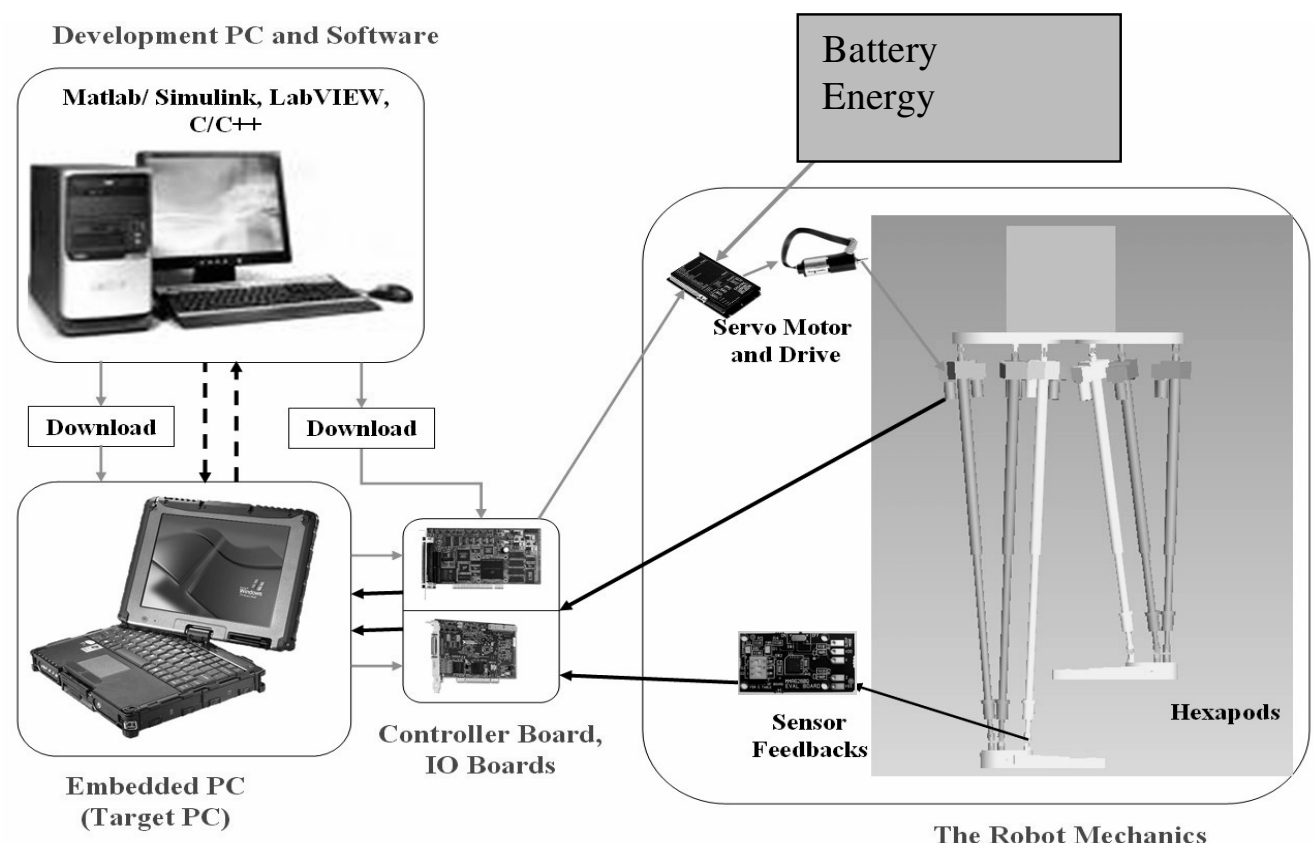

Fig. 6 The control system testing software/hardware tools configuration

Using the HiL method low-cost MEMS sensors can be bought and rapidly tested for their feasibility. This approach is efficient and very effective in step-by-step integration of control components as they arrive.

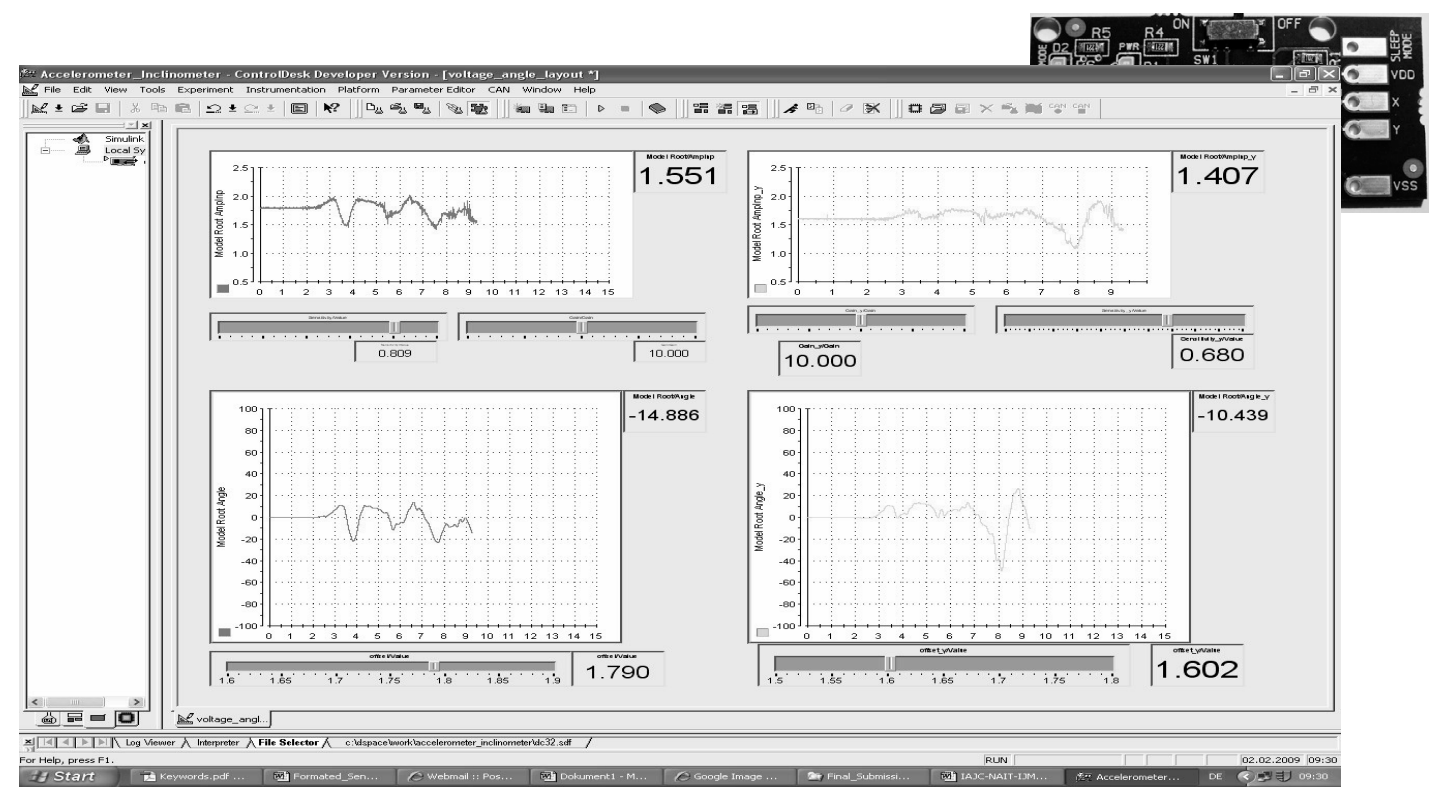

Fig. 7 Simulation of the roll and pitch orientation of a moving link using a MEMS based inclinomenter on dSPACE real time control board 


\section{Application areas}

Balance control motion sensors have very broad application. Accelerometers, inclinometers, and gyroscopes are broadly implemented in different balance/stability control application of mobile systems. Some examples of such mobile systems are active safety transportation and material handling vehicles, human body kinematics [9], biped robotics, online gaming industry [Nintendo Wii's sport games], and many other air, sea, and ground transportation vehicles.

\section{Conclusion}

Finding a fitting motion sensor solution for Centaurob's parallel kinematic applications with stringent geometric and precision requirements is challenging. Because of too many competing motion sensors in the market, it is easy to loose sight in the search process, if the selection process is not done in a systematic way. The variation in cost and quality can be huge. To avoid painful problems at later stage, it is recommended to make sensor design and/or selection in a systematic way. The application and solution requirement parameters have to be defined with detailed awareness. The study has explored different methods for computing positions and orientations (pose) of moving links in parallel mechanisms. In order to determine the pose, the deterministic nature of the forward kinematics of serial mechanisms is utilized by carefully selecting robot links to create a serial open loop. The discussed approach has used a minimum number of sensors to determine the pose of the robot. For developing and testing the selected sensor solutions, a PC-based hardware-in-the-loop (HiL) simulation method is being used. Here, Matlab/Simulink/Stateflow graphical development tools and dSPACE real time control system are used to interact with various sensors and actuators. This approach is effective in step-by-step integration of sensors and other hardware components as they arrive. In the poster presentation of the article, some of the simulation and prototyping results of the experimentation along with the hardware and software technologies used will be presented. Because of their small size, lower cost, and the technological trend mainly MEMS motion sensors are employed. MEMS sensors are not without limitation some of the limitations being lack of standardized packaging and complexity of their design and manufacturing process. All in all, however, MEMS based balance control motion sensors are very promising have a very broad application.

\section{References}

[1] J. Schlattmann. Laufmaschine und Verfahren zur Steuerung einer Laufmaschine. Deutsches Patent Nr. 19637501, 07/13/2000.

[2] J. Schlattmann, Mekonnen Tesfay Tesfu, Lars Ziemen. New Steps in the Development of the Two Legged Robot CENTAUROB. International Journal of Modern Engineering (IJME), Volume 7, Number 2, Spring 2007, USA.

[3] Mekonnen Tesfay Tesfu, J. Schlattmann, Lars Ziemen: Simulation Based Methodology for Selection and Integration of Real Time Control Electronics into Complex Dynamic Mechanical Systems: Proceedings of The 2008 IAJC-IJME International Conference - ISBN 978-1-60643379-9: 17-19 November, 2008, Nashville, USA

[4] Bob Scannell. Integrierte MEMS-Sensoren für Industriesteuerungen. Elektronik Praxis Nr.21, 7.November 2007.

[5] Mekonnen Tesfay Tesfu. Investigation of Optimization Strategy for Design Component-Selection. Master-Thesis, not published, Hamburg University of Technology, 2005.

[6] M. Meß, J. Schlattmann. Auswahl und Integration existierende elektronischer Komponenten mechatronischer Systeme am Beispiel von Sensoren. VDI-Berichte Nr. 1892, 2005.

[7] The MathWorks. http://www.mathworks.com/products/simulink/. June 2008.

[8] dSPACE Catalogue 2008.

[9] H. J. Luinge P.H. Veltink. Measuring orientation of human body segments using miniature gyroscopes and accelerometers. Signals Systems Group, Department of Electrical Engineering, University of Twente, Enschede, The Netherlands 